\title{
Comparison of different methods of determining $\beta$-lactam susceptibility in clinical strains of Pseudomonas aeruginosa
}

\author{
Eva Torres, Rosa Villanueva and Germán Bou \\ Servicio de Microbiología - Unidad de Investigación, Complejo Hospitalario Universitario Juan \\ Canalejo, La Coruña, Spain
}

Correspondence

Germán Bou

germanbou@canalejo.org

Received 3 August 2008

Accepted 12 January 2009

\section{INTRODUCTION}

Several automated systems are available for the identification and susceptibility testing of the most clinically important bacteria (Stager \& Davis, 1992). These systems are able to decrease the in-laboratory turnaround time required for standardized methods and to provide physicians with susceptibility profiles to help them select the most appropriate antimicrobial therapy (Felmingham \& Brown, 2001; Sanders et al., 2000). The reporting errors by any test systems can have serious implications for the clinical outcome for patients (Micek et al., 2005). Numerous studies have reported on the accuracy of various automated systems in testing several organismantimicrobial combinations. The most frequently reported errors have involved Pseudomonas aeruginosa and certain members of the family Enterobacteriaceae, especially when these organisms are tested against $\beta$-lactam antimicrobial agents (Biedenbach \& Jones, 1995; Biedenbach et al., 1999; Burns et al., 2001; Jones et al., 1998; Jones, 2001; Joyanes

Abbreviations: AES, Advanced Expert System; ATM, aztreonam; CAZ, ceftazidime; DD, disc diffusion; FEP, cefepime; IMP, imipenem; PIP, piperacillin; RM, reference method; TZP, piperacillin-tazobactam. et al., 2001; Karlowsky et al., 2003; Leverstein-van Hall et al., 2002; Ling et al., 2001; Saegeman et al., 2005; Steward et al., 2003; Sader et al., 2006; Juretschko et al., 2007). However, some of the automated systems exhibit a high, unacceptable level of very major (false susceptible) errors for some of the antibiotics tested with $P$. aeruginosa, thus emphasizing the need for manufacturers to re-evaluate these automated systems for testing of selected $\beta$-lactams with clinical isolates of P. aeruginosa (Sader et al., 2006).

As regards these automated systems, the ability of the VITEK 2 Advanced Expert System (AES) to identify antibiotic resistance phenotypes has been evaluated (Biedenbach \& Jones, 1995; Livermore et al., 2002). Although overall good results were obtained, the existence of some discrepancies suggests that certain modifications could be made to improve the performance of the system even further (Biedenbach \& Jones, 1995). Some of the mechanisms of resistance of $P$. aeruginosa to antimicrobial agents may preferentially affect $\beta$-lactam compounds, and some automated systems may not correct for this in the interpretation of the results (Jones et al., 1998; Karlowsky et al., 2003; Steward et al., 2003). Variations in inoculum concentrations and incubation times may also affect the 
detection of $\beta$-lactam resistance in $P$. aeruginosa. Thus rapid automated susceptibility testing systems may be poor at detecting resistance to some $\beta$-lactam compounds for technical reasons (including the methodologies used by the test system and software calculations), because of the underlying resistance mechanisms of the organism (Biedenbach \& Jones, 1995; Biedenbach et al., 1999; Doern et al., 1997). The presence of spontaneous $\beta$ lactam-resistant revertants (or mutants), which may appear as isolated colonies on the surface of the agar plate and may be selected during incubation with the antibiotic, may not be detected by a microdilution or automated method.

In the present study, we evaluated the VITEK 2 system and two non-automated methods, agar disc diffusion (DD) and Etest, for testing the susceptibility of $101 P$. aeruginosa isolates to six $\beta$-lactams: piperacillin (PIP), piperacillintazobactam (TZP), ceftazidime (CAZ), cefepime (FEP), aztreonam (ATM) and imipenem (IMP). The results were compared with those obtained by the standard method recommended by the CLSI, i.e. broth microdilution. We also assessed how the AES used with the VITEK 2 system interprets MICs of antibiotics tested with $P$. aeruginosa clinical isolates, with some modifications for interpreting $\beta$-lactam-resistant phenotypes in $P$. aeruginosa.

\section{METHODS}

Bacterial strains. A total of 101 clinical isolates of P. aeruginosa collected (between 2003 and 2005) from the Juan Canalejo Hospital, NW Spain, were included in the study. Isolates were identified by API 20NE (bioMérieux). Only one strain per patient was selected. In some cases and to discount clonality, molecular typing was performed by use of the Ready-To-Go-RAPD Analysis kit (GE Healthcare). P. aeruginosa ATCC 27853 was used as a quality control in antibiotic susceptibility determinations.

Antimicrobial susceptibility testing. Broth microdilution (reference method, RM) and DD were performed according to CLSI criteria (CLSI, 2007; NCCLS, 1997). Susceptibility tests with the VITEK 2 system were performed with the AST-N022 card (bioMérieux Vitek) according to the manufacturer's instructions. Etest ( $\mathrm{AB}$ Biodisk) was also carried out according to the manufacturer's instructions. The following antimicrobial agents were tested: PIP, CAZ and ATM (Sigma-Aldrich), TZP (Wyeth-Ayerst Laboratories), FEP (Bristol-Myers Squibb) and IMP (MSD, Merck Sharp \& Dohme). The MIC values corresponded to three independent determinations. To be considered valid, identical MIC values had to be obtained in at least two determinations, otherwise experiments were repeated.

VITEK 2 AES. As no specific rules for $\beta$-lactam resistance (Quale et al., 2006) (interplay between AmpC hyperproduction, several efflux systems and $o p r D$ production) in P. aeruginosa are included in the AES, some new ones were included and taken into consideration for interpretation of $\beta$-lactam MICs related to AmpC hyperproduction: (i) if the MIC of ticarcillin was higher than $256 \mathrm{mg} \mathrm{l}^{-1}$ then the bacterial isolates were classified by the AES as resistant to PIP and $\mathrm{TZP}$, independently of the corresponding MIC values; (ii) if the isolates were resistant to PIP and CAZ, then they were also assumed to be resistant to ticarcillin, ticarcillin-clavulanic acid and TZP, and to show at least intermediate resistance to FEP and ATM, even if the bacterial isolate showed full susceptibility to these antibiotics; (iii) if the MIC of CAZ was higher than $16 \mathrm{mg} \mathrm{l}^{-1}$, then even if the bacterial isolates showed full susceptibility to these antibiotics, they were considered to be resistant to FEP and ATM; and (iv) if resistance to CAZ, ATM and FEP was intermediate or full, the bacterial isolate was considered resistant to TZP, independent of the corresponding MIC value. For imipenem, no rules for interpretation of MICs were taken into consideration.

Analysis of results. Very major errors were considered when an organism was defined as resistant by the RM (broth microdilution) but was categorized as susceptible by an alternative method. Major errors were defined when an organism found to be susceptible by the RM was categorized as resistant by an alternative method. Minor errors occurred when an organism was considered susceptible or resistant by the RM, but intermediate by another method, and/or when it showed intermediate resistance by the RM but was susceptible or resistant by the other methods.

Essential agreement was used to compare MICs obtained by the RM with those obtained by the remaining methods and was considered to occur when the MICs (between pairs) were within one twofold dilution.

Agreement with clinical category was used to compare antibiotic resistance categorization by consideration of two different categories, susceptible and intermediate/resistant (which for practical reasons were grouped in the same category).

\section{RESULTS AND DISCUSSION}

One hundred and one clinical isolates of $P$. aeruginosa were analysed by broth microdilution, automated VITEK 2, Etest and DD.

The distribution of the MIC results for $P$. aeruginosa bacterial isolates by broth microdilution, VITEK 2 and Etest are shown in Table 1 . The $\mathrm{MIC}_{50}$ and $\mathrm{MIC}_{90}$ values for these methods are shown in Table 2. Overall, the Etest results almost fully correlated with the $\mathrm{MIC}_{90}$ values obtained by the RM. However VITEK 2 yielded lower $\mathrm{MIC}_{50}$ and $\mathrm{MIC}_{90}$ values with six $\beta$-lactam antibiotics tested (Table 2).

On the basis of these results, the rates of susceptibility and resistance obtained by the RM are shown in Table 3. The rates of susceptibility category for DD with the same bacterial isolates were very similar to these values. With FEP, the rate of resistance to this antibiotic fell to $51.48 \%$, although the higher value in the intermediate category compensated for this difference, and indeed the rate of susceptibility was $22.77 \%$, which is quite similar to that by the RM.

With VITEK 2, differences in the rate of resistance were observed for CAZ, FEP, ATM and IMP as compared with the RM (Table 3). It is important to point out that although VITEK 2 yielded lower MIC values for some of these antibiotics (see Tables 1 and 2), the antibiotic susceptibility was correctly assigned by the AES (Table 3 ). The rates of antibiotic susceptibility obtained by VITEK 2 were very similar to those obtained by the RM (except for TZP). The rates of resistance were lower (trend to false susceptibility) than those obtained by the RM. However, 
Table 1. MICs $\left(\mathrm{mg} \mathrm{l}^{-1}\right)$ of the antibiotics tested with the challenge collection of $P$. aeruginosa strains $(n=101)$, obtained by broth microdilution (RM), VITEK 2 and Etest

Values are the number of strains for which the particular MIC was obtained.

\begin{tabular}{|c|c|c|c|c|c|c|c|c|c|}
\hline \multirow[t]{2}{*}{ Antibiotic } & \multicolumn{9}{|c|}{$\operatorname{MIC}\left(\mathrm{mg} \mathrm{l}^{-1}\right)$} \\
\hline & $\leqslant 1$ & 2 & 4 & 8 & 16 & 32 & 64 & 128 & $\geqslant 256$ \\
\hline \multicolumn{10}{|c|}{ Broth microdilution } \\
\hline PIP & 0 & 1 & 2 & 0 & 3 & 1 & $5^{\star}$ & $3 \dagger$ & 86 \\
\hline TZP & 0 & 2 & 3 & 4 & 2 & 7 & $5^{*}$ & $16 \dagger$ & 62 \\
\hline CAZ & 0 & 6 & 4 & $5^{*}$ & 7 & $9 \dagger$ & 40 & 19 & 11 \\
\hline FEP & 0 & 5 & 3 & $9^{*}$ & 8 & $26 \dagger$ & 23 & 8 & 19 \\
\hline ATM & 0 & 2 & 4 & $7^{\star}$ & 33 & $22 \dagger$ & 19 & 6 & 8 \\
\hline IMP & 1 & 1 & $6^{*}$ & 2 & $34 \dagger$ & 57 & NT & NT & NT \\
\hline \multicolumn{10}{|l|}{ VITEK 2} \\
\hline PIP & 0 & 1 & 6 & 7 & 3 & 11 & $22^{*}$ & $51 \dagger$ & NT \\
\hline TZP & 0 & 1 & 6 & 9 & 1 & 17 & $34^{*}$ & $33 \dagger$ & NT \\
\hline CAZ & 6 & 2 & 7 & $0^{*}$ & 40 & $6 \dagger$ & 40 & NT & NT \\
\hline FEP & 4 & 9 & 7 & $21^{*}$ & 38 & $9 \dagger$ & 12 & NT & NT \\
\hline ATM & 3 & 7 & 4 & $2^{*}$ & 44 & $12 \dagger$ & 29 & NT & NT \\
\hline IMP & 7 & 7 & $5^{\star}$ & 8 & $74 \dagger$ & NT & NT & NT & NT \\
\hline \multicolumn{10}{|l|}{ Etest } \\
\hline PIP & 1 & 0 & 3 & 1 & 5 & 1 & $0^{*}$ & $2 \dagger$ & 88 \\
\hline TZP & 1 & 0 & 2 & 4 & 2 & 1 & $1^{*}$ & $2 \dagger$ & 87 \\
\hline CAZ & 0 & 4 & 4 & $2^{*}$ & 2 & $9 \dagger$ & 16 & 10 & 53 \\
\hline FEP & 0 & 2 & 1 & $6^{*}$ & 10 & $11 \dagger$ & 26 & 19 & 26 \\
\hline ATM & 3 & 5 & 7 & $3^{\star}$ & 18 & $28 \dagger$ & 19 & 5 & 13 \\
\hline IMP & 1 & 4 & $6^{*}$ & 2 & $2 \dagger$ & 86 & NT & NT & NT \\
\hline
\end{tabular}

NT, Concentration not tested.

*Susceptibility breakpoint.

$†$ †esistance breakpoint.

the number of isolates belonging to the intermediate category compensated for the low number of resistant isolates, and when the intermediate and resistant categories were considered together, the percentages were very similar to those yielded by the RM.

With Etest, the rates of resistance of the isolates were slightly higher with TZP and CAZ (trend to false

Table 2. $\mathrm{MIC}_{50}$ and $\mathrm{MIC}_{90}\left(\mathrm{mg} \mathrm{l}^{-1}\right)$ values for antimicrobials tested by broth microdilution (RM), VITEK 2 and Etest

\begin{tabular}{|lllrrrrr|}
\hline & Method & PIP & TZP & CAZ & FEP & ATM & IMP \\
\hline \multirow{2}{*}{ MIC $90_{90}$} & RM & 256 & 256 & 256 & 256 & 128 & 32 \\
& Etest & 256 & 256 & 256 & 256 & 256 & 32 \\
& VITEK 2 & 128 & 128 & 64 & 64 & 64 & 16 \\
MIC $_{50}$ & RM & 256 & 256 & 64 & 32 & 32 & 32 \\
& Etest & 256 & 256 & 192 & 64 & 32 & 32 \\
& VITEK 2 & 128 & 64 & 16 & 16 & 16 & 16 \\
\hline
\end{tabular}

resistance) but almost identical with PIP, FEP, ATM and IMP.

Categorization results were initially obtained by comparing the results of the RM with those obtained by the automated method, Etest and DD (Table 4).

The greatest numbers of inter-method disagreements corresponded to TZP, for which a large number of major errors were obtained with the three methods under evaluation, 11.88, 10.89 and $5.94 \%$ for VITEK 2, Etest and DD (tendency towards false-resistance results). There was a significant number of very major errors (trend to false-susceptible results) with FEP and ATM by DD. A high percentage of minor errors was obtained with CAZ, FEP and ATM by all three methods studied, although particularly with VITEK 2 (trend to false susceptibility; Table 3). Of note was the high percentage of very major errors with VITEK 2 and IMP.

Overall a good essential agreement was obtained with the six antibiotics studied, with the exception of PIP, TZP and FEP by VITEK 2 (trend to false susceptibility). Also of note was the low essential agreement with CAZ by Etest. As regards agreement with clinical category, the three methods yielded good agreement with the RM (mostly higher than $90 \%)$ for all six $\beta$-lactam antibiotics studied.

The main objective of the present study was to compare three different methods (VITEK 2, Etest and DD) for monitoring antibiotic susceptibility in clinical isolates of $P$. aeruginosa. The main mechanisms involved in $\beta$-lactam resistance in $P$. aeruginosa are AmpC hyperproduction, acquisition of $\beta$-lactamases, loss or reduction of porins and expression of efflux pumps (Livermore, 2001). Any of these different mechanisms or any combination of them produces complex profiles of antibiotic susceptibility phenotypes, making it very difficult to predict the effectiveness of the different $\beta$-lactam antibiotics. In this regard, correct interpretation of the MIC data is key in defining the antibiotic susceptibility profile in order to select the most appropriate antibiotherapy.

The data analysis presented here showed good agreement among methods. However, although the results obtained by Etest, DD and VITEK 2 correlated well with those obtained by RM for those $P$. aeruginosa isolates that yielded the highest or lowest MIC values (clearly resistant or clearly susceptible), for the bacterial isolates that yielded intermediate MIC values (and some of them with mutant subpopulations) such good correlations were not obtained (data not shown), resulting in a lack of overall good correlation. In this regard, it is important to mention that in this study CAZ discs and Etest was the best combination of antibiotic and test to detect heterogeneous populations (data not shown).

It was also important to implement rules for interpreting MIC values to determine $\beta$-lactam resistance in $P$. aeruginosa with the AES. The modifications in MIC interpretation allowed us to establish a new pattern in 
Table 3. Susceptibility category by the three methods evaluated and broth microdilution (RM)

\begin{tabular}{|lcccc|}
\hline $\begin{array}{l}\text { Antimicrobial } \\
\text { and category }\end{array}$ & $\begin{array}{c}\text { Reference } \\
\text { method (\%) }\end{array}$ & $\begin{array}{r}\text { DD } \\
(\%)\end{array}$ & $\begin{array}{c}\text { VITEK 2 } \\
(\%)\end{array}$ & $\begin{array}{c}\text { Etest } \\
(\%)\end{array}$ \\
\hline PIP & & & & \\
Susceptible & 11.88 & 12.87 & 10.89 & 11.88 \\
Resistant & 88.12 & 87.13 & 89.11 & 88.12 \\
TZP & & & & \\
Susceptible & 22.77 & 19.8 & 10.89 & 11.88 \\
Resistant & 77.23 & 80.2 & 89.11 & 88.12 \\
CAZ & & & & \\
Susceptible & 14.85 & 15.84 & 13.86 & 9.9 \\
Intermediate & 6.93 & 22.77 & 40.59 & 4.95 \\
Resistant & 78.21 & 71.29 & 45.54 & 85.15 \\
FEP & & & & \\
Susceptible & 16.8 & 22.77 & 13.86 & 8.9 \\
Intermediate & 7.9 & 25.74 & 62.38 & 14.85 \\
Resistant & 75.3 & 51.48 & 23.76 & 76.24 \\
ATM & & & & \\
Susceptible & 12.87 & 19.8 & 10.89 & 17.82 \\
Intermediate & 32.67 & 29.7 & 50.49 & 28.71 \\
Resistant & 54.46 & 50.5 & 38.61 & 53.47 \\
IMP & & & & \\
Susceptible & 7.92 & 16.83 & 18.81 & 10.89 \\
Intermediate & 1.98 & 1.98 & 7.92 & 1.98 \\
Resistant & 90.1 & 81.19 & 73.27 & 87.13 \\
& & & & \\
\hline
\end{tabular}

the antibiotic susceptibility of the $P$. aeruginosa population beyond the absolute MIC values. Thus although VITEK 2 generally produced lower MIC values than the RM, for most of the $\beta$-lactam antibiotics the AES allowed a good categorization of the $P$. aeruginosa isolates in terms of $\beta$ lactam resistance phenotypes.

Among the studied methods, Etest yielded the highest number of false-resistance results with all antibiotics tested, whereas DD yielded the highest number of false-susceptible results with all antibiotics except CAZ. VITEK 2 yielded the highest number of minor errors but maintained good agreement with clinical category.

It is important to note that most of the lack of concordance with VITEK 2 corresponded to isolates assigned to the intermediate category, and in these cases it may be useful to apply an alternative method (such as Etest) to confirm MIC values.

The results presented here are similar to those reported by Joyanes et al. (2001), who evaluated the VITEK 2 system for susceptibility testing of $P$. aeruginosa clinical isolates by comparing data with those obtained by the microdilution RM. However, these authors tested strains with clinically defined categories of phenotypes of resistance to CAZ, ticarcillin, IMP, meropenem and ciprofloxacin. It should be noted that the distribution of phenotypes among the strains tested in that study (Joyanes et al., 2001) does not reflect the distribution of phenotypes among routine clinical isolates. By contrast, the strains used in the present
Table 4. Essential agreement (EA), agreement with clinical category (ACC) and errors in clinical categories between VITEK 2, Etest, DD and the RM

\begin{tabular}{|lccc|}
\hline Antibiotic & VITEK 2 (\%) & Etest $(\%)$ & DD $(\%)$ \\
\hline PIP & & & \\
Very major error & 0 & 0 & 0.99 \\
Major error & 0.99 & 0.99 & 0.99 \\
EA & 79.21 & 94.06 & - \\
ACC & 99.0 & 99.0 & 98.02 \\
TZP & & & \\
Very major error & 0 & 0 & 0.99 \\
Major error & 11.88 & 10.89 & 5.94 \\
EA & 84.16 & 92.08 & - \\
ACC & 98.02 & 89.11 & 93.07 \\
CAZ & & & \\
Very major error & 0.99 & 0 & 0 \\
Major error & 0 & 4.95 & 0 \\
Minor error & 39.60 & 9.9 & 22.77 \\
EA & 89.11 & 86.14 & - \\
ACC & 95.04 & 93.07 & 99.01 \\
FEP & & & \\
Very major error & 0.99 & 0 & 4.95 \\
Major error & 0 & 1.98 & 0 \\
Minor error & 58.42 & 16.83 & 28.71 \\
EA & 71.29 & 90.1 & - \\
ACC & 94.06 & 91.09 & 91.09 \\
ATM & & & \\
Very major error & 0 & 0 & 2.97 \\
Major error & 0 & 0 & 0 \\
Minor error & 36.63 & 30.69 & 30.69 \\
EA & 97.03 & 89.11 & - \\
ACC & 91.09 & 96.03 & 93.07 \\
IMP & & & \\
Very major error & 8.9 & 0.99 & 6.93 \\
Major error & 0 & 0 & 0 \\
Minor error & 9.9 & 3.96 & 3.96 \\
EA & 92.08 & 100 & - \\
ACC & & & \\
& & & \\
\hline
\end{tabular}

study represent phenotypes isolated during routine clinical practice in the Juan Canalejo hospital, as we included isolates that yielded high, intermediate and low $\beta$-lactam MIC values.

In summary, and considering some of the limitations described above, VITEK 2 with an improved AES for interpreting $\beta$-lactam resistance phenotypes in $P$. aeruginosa is an appropriate alternative method for determining $\beta$-lactam MIC values for this micro-organism, especially with isolates that overexpress the AmpC enzyme (reflected by high cephalosporin MIC values). Nevertheless, it is important to consider that with $P$. aeruginosa isolates that yield intermediate CAZ, ATM or FEP MICs, or with strains that display a lack of correlation in categorization (i.e. susceptible to one antibiotic, CAZ, but resistant to the remaining two, ATM and FEP), a diffusion method (such as Etest) must be carried out in addition to VITEK 2, to 
increase the accuracy of determination of MIC values and categorization of antibiotics.

\section{ACKNOWLEDGEMENTS}

The study was partly financed by the Consellería de Innovación, Industria y Comercio, Xunta de Galicia (PGIDIT04BTF916028PR), Fondo de Investigaciones Sanitarias (PI061368, PI081613), and also supported by the Ministerio de Sanidad y Consumo, ISCIII, Spanish Network for the Research in Infectious Diseases (REIPI RD06/0008).

\section{REFERENCES}

Biedenbach, D. J. \& Jones, R. N. (1995). Interpretive errors using an automated system for the susceptibility testing of imipenem and aztreonam. Diagn Microbiol Infect Dis 21, 57-60.

Biedenbach, D. J., Marshal, S. A. \& Jones, R. N. (1999). Accuracy of cefepime antimicrobial susceptibility testing results for Pseudomonas aeruginosa tested on the MicroScan WalkAway system. Diagn Microbiol Infect Dis 33, 305-307.

Burns, J. L., Saiman, L., Whittier, S., Krzewinski, J., Liu, Z., Larone, D. Marshall, S. A. \& Jones, R. N. (2001). Comparison of two commercial systems (Vitek and MicroScan-WalkAway) for antimicrobial susceptibility testing of Pseudomonas aeruginosa isolates from cystic fibrosis patients. Diagn Microbiol Infect Dis 39, 257-260.

CLSI (2007). Performance Standards for Antimicrobial Susceptibility Testing. Seventeenth Informational Supplement. M100-S17. Wayne, PA: Clinical and Laboratory Standards Institute.

Doern, G. V., Brueggemann, A. B., Perla, R., Daly, J., Halkias, D., Jones, R. N. \& Sanbolle, M. A. (1997). Multicenter laboratory evaluation of the bioMerieux Vitek antimicrobial susceptibility testing system with 11 antimicrobial agents versus members of the family Enterobacteriaceae and Pseudomonas aeruginosa. J Clin Microbiol 35, 2115-2119.

Felmingham, D. \& Brown, D. F. J. (2001). Instrumentation in antimicrobial susceptibility testing. J Antimicrob Chemother 48 (Suppl. 1), 81-85.

Jones, R. N. (2001). Method preferences and test accuracy of antimicrobial susceptibility testing. Arch Pathol Lab Med 125, 1285-1289.

Jones, R. N., Biedenbach, D. J., Marshall, S. A., Pfaller, M. A. \& Doern, G. V. (1998). Evaluation of the Vitek system to accurately test the susceptibility of Pseudomonas aeruginosa clinical isolates against cefepime. Diagn Microbiol Infect Dis 32, 107-110.

Joyanes, P., Conejo, M. C., Martinez-Martinez, L. \& Perea, E. J. (2001). Evaluation of the VITEK 2 system for the identification and susceptibility testing of three species of nonfermenting gram-negative rods frequently isolated from clinical samples. J Clin Microbiol 39, 3247-3253.

Juretschko, S., LaBombardi, V. J., Lerner, S.,Schreckenberger, P. C. $\&$ the Pseudomonas AST Study Group. (2007). Accuracies of $\beta$ lactam susceptibility test results for Pseudomonas aeruginosa with four automated systems (BD Phoenix, MicroScan, WalkAway, and Vitek 2). J Clin Microbiol 45, 1339-1342.
Karlowsky, J. A., Weaver, M. K., Thornsberry, C., Dowzicky, M. J., Jones, M. E. \& Sahm, D. F. (2003). Comparison of four antimicrobial susceptibility testing methods to determine the in vitro activities of piperacillin and piperacillin-tazobactam against clinical isolates of Enterobacteriaceae and Pseudomonas aeruginosa. J Clin Microbiol 41, 3339-3343.

Leverstein-van Hall, M. A., Fluit Paaum, A., Box, A. T. A., Brisse, S. \& Verhoef, J. (2002). Evaluation of the Etest ESBL and the BD Phoenix, VITEK 1, and VITEK 2 automated instruments for detection of extended-spectrum beta-lactamases in multiresistant Escherichia coli and Klebsiella spp. J Clin Microbiol 40, 3703-3711.

Ling, T. K. W., Tam, P. C., Liu, Z. K. \& Cheng, A. F. B. (2001). Evaluation of VITEK 2 rapid identification and susceptibility testing system against gram-negative clinical isolates. J Clin Microbiol 39, 2964-2966.

Livermore, D. M. (2001). Of Pseudomonas, porins, pumps and carbapenems. J Antimicrob Chemother 47, 247-250.

Livermore, D. M., Struelens, M., Amorim, J., Baquero, F., Bille, J., Canton, R., Henning, S., Gatermann, S., Marchese, A. \& other authors (2002). Multicentre evaluation of the VITEK2 advanced expert system for interpretative reading of antimicrobial resistance tests. J Antimicrob Chemother 49, 289-300.

Micek, S. T., Lloyd, A. E., Ritchie, D., Reichley, R. M., Fraser, V. J. \& Kollef, M. H. (2005). Pseudomonas aeruginosa bloodstream infection: importance of appropriate initial antimicrobial treatment. Antimicrob Agents Chemother 49, 1306-1311.

NCCLS (1997). Performance Standards for Antimicrobial Disk Susceptibility Tests. Approved Standard M2-A6. Wayne, PA: National Committee for Clinical Laboratory Standards.

Quale, J., Bratu, S., Gupta, J. \& Landman, D. (2006). Interplay of efflux system, $a m p C$, and $o p r D$ expression in carbapenem resistance of Pseudomonas aeruginosa clinical isolates. Antimicrob Agents Chemother 50, 1633-1641.

Sader, H. S., Fritsche, T. R. \& Jones, R. N. (2006). Accuracy of three automated systems (MicroScan WalkAway, VITEK, and VITEK 2) for susceptibility testing of Pseudomonas aeruginosa against five broadspectrum beta-lactam agents. J Clin Microbiol 44, 1101-1104.

Saegeman, V., Huynen, P., Colaert, J., Melin, P. \& Verhaegen, J. (2005). Susceptibility testing of Pseudomonas aeruginosa by the Vitek 2 system: a comparison with E-test results. Acta Clin Belg 60, 3-9.

Sanders, C. C., Peyret, M., Smith Moland, E., Shubert, C., Thomson, K. S., Boeufgras, J. M. \& Sanders, W. E., Jr (2000). Ability of the VITEK 2 Advanced Expert System to identify $\beta$-lactam phenotypes in isolates of Enterobacteriaceae and Pseudomonas aeruginosa. J Clin Microbiol 38, 570-574.

Stager, C. E. \& Davis, J. R. (1992). Automated systems for the identification of microorganisms. Clin Microbiol Rev 5, 302-327.

Steward, C. D., Mohammed, J. M., Swenson, J. M., Stocker, S. A., Williams, P. P., Gaynes, R. P., McGowan, J. E., Jr \& Tenover, F. C. (2003). Antimicrobial susceptibility testing of carbapenems: multicenter validity testing and accuracy levels of five antimicrobial test methods for detecting resistance in Enterobacteriaceae and Pseudomonas aeruginosa isolates. J Clin Microbiol 41, 351-358. 